\title{
REA_11 - Low-cost protocol for rapid detection of ZIKV from patient and mosquito samples using a direct-RT-qPCR assay without RNA extraction step
}

Severino Jefferson Ribeiro da Silva ${ }^{1 *}$; Renata Pessôa Germano Mendes $^{1}$; Jurandy Júnior Ferraz de Magalhães $^{2}$; Elisa de Almeida Neves Azevedo ${ }^{1}$; Marília de Albuquerque Sena ${ }^{1}$; Bárbara Nazly Rodrigues Santos $^{1}$; Caroline Targino Alves da Silva ${ }^{1}$; Keith Pardee ${ }^{3}$; Lindomar Pena ${ }^{1}$.

${ }^{1}$ Fiocruz - Fundação Oswaldo Cruz;

${ }^{2}$ LACEN-PE;

${ }^{3}$ University of Toronto.

Introduction: The recent epidemic of the Zika virus (ZIKV) in the Americas transformed the virus into a global concern, since infection is associated with the development of congenital neurological disease. The reverse transcriptase reaction followed by quantitative polymerase chain reaction (RT-qPCR) is considered the gold standard molecular method to diagnose ZIKV-infection. However, the time- and labor-intensive sample preparation and the RNA extraction using commercial kits are required to remove inhibitors that cause false-negative results. These drawbacks associated with high cost of extraction kits greatly limit the diagnosis with a large number of samples, especially in remote areas during epidemic scenarios. Moreover, the COVID-19 pandemic has worsened this situation since most diagnostic resources and supplies have been directed for SARS-CoV-2. Therefore, methodological simplification could increase diagnostic availability and efficiency, benefitting patient care.

Objective: In this context, the aim of this work was to optimize and validate an alternative protocol for RNA extraction based on a boiling water bath coupled with the RT-qPCR for rapid and direct detection of ZIKV in patient and mosquito samples.

Methodology: In all experiments, the ZIKV strain named PE243/2015 was used. Initially, the temperature and time of boiling was optimized in order to determine the better combination with great performance when compared to reference method using RNA extraction commercial kit. In addition, we confirmed effectiveness of heat inactivation by plaque assay. Then, we determined the capacity of RT-qPCR to detect ZIKV in human biological samples (serum, urine, saliva and semen) and mosquito samples under controlled conditions. The analytical specificity and analytical sensitivity of the protocol were evaluated. Finally, we validated the RT-qPCR protocol without RNA extraction using 205 clinical samples collected at the epicenter of the last Zika epidemic in Latin America.

Results: Using human biological samples including serum, urine, saliva and semen or mosquito samples infected with ZIKV under controlled conditions, we show analytical specificity and sensitivity equivalent to the RT-qPCR using extraction kit for ZIKV detection. The RT-qPCR without RNA extraction had a sensitivity of $86.67 \%$, specificity of $99.38 \%$, and overall accuracy of $96.59 \%$, highlighting the potential of our alternative protocol for detection of ZIKV without RNA extraction in patient and mosquito samples. The alternative protocol reported here allows for the direct use of patient (serum, urine, saliva and semen) or mosquito samples in just 2 hours since sample collection until release of results, instead of 5 hours in the standard procedure.

Conclusion: This robust direct-RT-qPCR assay shows a great potential for use in low-resource settings, particularly in developing countries where ZIKV is endemic. Our low-cost protocol it realizes the goal of large-scale on-site screening for ZIKV infection and could be used for early diagnosis and the prevention of future outbreaks. increase the diagnostic capacity of ZIKV-affected low-resource scenarios

Keywords: Diagnostic; Low cost; Zika 